\title{
Settlement analysis of viscoelastic foundation under vertical line load using a fractional Kelvin-Voigt model
}

\author{
Hong-Hu Zhu*1, Lin-Chao Liu ${ }^{2}$, Hua-Fu Pei ${ }^{3}$ and Bin Shi ${ }^{1}$ \\ ${ }^{1}$ School of Earth Sciences and Engineering, Nanjing University, Nanjing, China \\ ${ }^{2}$ School of Civil Engineering, Xinyang Normal University, Xinyang, China \\ ${ }^{3}$ Department of Civil and Structural Engineering, The Hong Kong Polytechnic University, Hong Kong, China
}

(Received January 23, 2011, Revised February 19, 2012, Accepted March 05, 2012)

\begin{abstract}
Soil foundations exhibit significant creeping deformation, which may result in excessive settlement and failure of superstructures. Based on the theory of viscoelasticity and fractional calculus, a fractional Kelvin-Voigt model is proposed to account for the time-dependent behavior of soil foundation under vertical line load. Analytical solution of settlements in the foundation was derived using Laplace transforms. The influence of the model parameters on the time-dependent settlement is studied through a parametric study. Results indicate that the settlement-time relationship can be accurately captured by varying values of the fractional order of differential operator and the coefficient of viscosity. In comparison with the classical Kelvin-Voigt model, the fractional model can provide a more accurate prediction of long-term settlements of soil foundation. The determination of influential distance also affects the calculation of settlements.
\end{abstract}

Keywords: soil foundation; fractional viscoelastic model; the Flamant-Boussinesq solution; settlement; Laplace transform.

\section{Introduction}

Research results show that the soil foundation exhibits significant creeping behaviour and the ground deformation under loading from superstructures is highly time-dependent (Bjerrum 1967). For the estimation of the increase in stresses at various points and associated displacements caused in soil foundations due to external loading, there have been a number of viscoelastic, elastic-viscoplastic (EVP) or elastoplastic-viscoplastic models (e.g., Christie 1964, Kaliakin and Dafalias 1990, Yin and Graham 1994, Justo and Durand 2000). Among them, viscoelastic foundation models can provide satisfactory simulation of the time-dependent behaviour when the stress level in soil is low and yielding of soil is thus not reached. The ideal assumption of the theory of viscoelasticity, namely that the foundation is homogeneous, viscoelastic, and isotropic, is not quite true in most cases. However, it provides a close estimation of long-term settlement and failure possibility of superstructures and, using proper safety factors, a safe design can be developed.

Because integer-order differential operators are used in the viscoelastic constitutive equations, the

*Corresponding author, Associate Professor, E-mail: zhh@nju.edu.cn 
kernel functions (creep modulus and relaxation modulus) of viscoelastic models are normally a combination of exponential functions. Thus these models have difficulties in predicting settlement of soil foundation accurately and their applicable range is significantly limited. Fractional constitutive models of viscoelastic materials were first introduced by Gemant (1936). In the constitutive equations, integer-order differential operators were replaced by fractional-order differential operators. These models have shown powerful capability in capturing static and dynamic stress-strain-time relationships of viscoelastic materials and have been applied in various fields in the past few decades (Bagley and Torvik 1983 and 1986, Welch et al. 1999, Zhang and Shimizu 1998, Li et al. 2001, Rossikhin and Shitikova 2010). However, due to the complexity involved in obtaining the analytical solution, these fractional models have not been applied in the field of geotechnical engineering until recent years (Atanackovic and Stankovic 2004, Dikmen 2005, Liu et al. 2006, Paola et al. 2009, Zhu et al. 2011).

In this paper, a fractional Kelvin-Voigt viscoelastic constitutive model was proposed to study timedependent settlement of soil foundations. The analytical solution of a half-space under a vertical line load is derived using Laplace transforms. The influences of the model parameters and the predetermined influential distance on the calculated settlements at the foundation surface are further explored using a parametric study.

\section{Fractional Kelvin-Voigt model}

The generalized viscoelastic constitutive equation using fractional derivatives can be described by (Padovan 1987)

$$
\sigma(t)+\sum_{m=1}^{M} b_{m} \frac{d^{\beta_{m}}}{d t^{\beta_{m}}} \sigma(t)=E_{0} \varepsilon(t)+\sum_{n=1}^{N} E_{n} \frac{d^{\alpha_{n}}}{d t^{\alpha_{n}}} \varepsilon(t)\left(0<\beta_{m}<1,0<\alpha_{n}<1\right)
$$

where $m$ and $n$ are positive integers; $d^{\beta_{m}} / d t^{\beta_{m}}$ and $d^{\alpha_{n}} / d t^{\alpha_{n}}$ are Riemann-Liouville fractional differential operators defined by (Miller and Ross 1993)

$$
\frac{d^{\alpha} \mathrm{f}(t)}{d t^{\alpha}}=\frac{1}{\Gamma(1-\alpha)} \frac{\mathrm{d}}{\mathrm{d} t} \int_{0}^{t} \frac{\mathrm{f}(\tau)}{(t-\tau)^{\alpha}} \mathrm{d} \tau(0<\alpha<1)
$$

where $\Gamma(u)$ is the Gamma function, $\Gamma(u)=\int_{0}^{\infty} t^{u-1} e^{-u} d t$.

If $m=n=1$ and $b_{1}=0$, Eq. (1) can be expressed by

$$
\sigma(t)=E_{0} \varepsilon(t)+E_{1} \frac{d^{\alpha} \varepsilon(t)}{d t^{\alpha}}
$$

It is obvious that if $\alpha=1$, Eq. (3) represents the constitutive equation of the classical Kelvin-Voigt viscoelastic model (KVM). Thus this new model can be called a generalized fractional Kelvin-Voigt model (FKVM), in which the integer-order differential operator is now replaced by a fractionalorder one.

In the theory of viscoelasticity, the stress-strain relationships are

$$
P^{\prime} S_{i j}(t)=Q^{\prime} e_{i j}(t)
$$




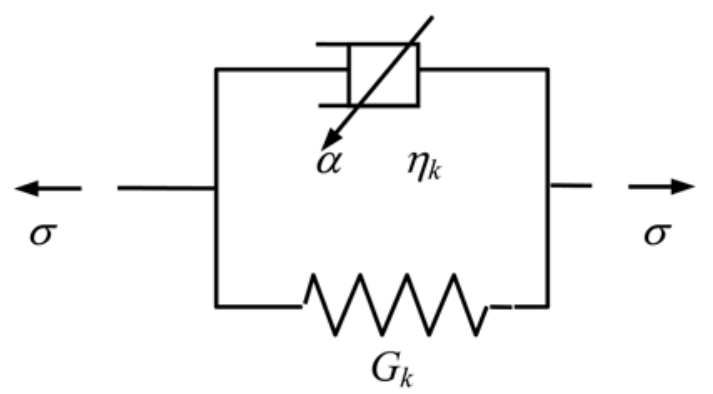

Fig. 1 Three-parameter fractional Kelvin-Voigt model

$$
P^{\prime \prime} \sigma_{i j}(t)=Q^{\prime \prime} \varepsilon_{i j}(t)
$$

where $S_{i j}(t)$ and $\sigma_{i j}(t)$ are deviatoric and hydrostatic stress tensors; $e_{i j}(t)$ and $\varepsilon_{i j}(t)$ are deviatoric and hydrostatic strain tensors; $P^{\prime}, Q^{\prime}, P^{\prime \prime}$ and $Q^{\prime \prime}$ are linear differential operators consisting of viscoelastic parameters.

The volumetric and shear strains of foundation soils under low stress levels are assumed to be time-dependent. The FKVM shown in Fig. 1 is thus utilized for the description of the viscoelastic behaviour of the bulk and shear moduli,, which describe the volume and shape changes of soil mass under external loading. Thus

$$
\begin{gathered}
P^{\prime}=1 \\
Q^{\prime}=G+\eta \frac{d^{\alpha}}{d t^{\alpha}} \\
P^{\prime \prime}=1 \\
Q^{\prime \prime}=K+\eta \frac{d^{\alpha}}{d t^{\alpha}}
\end{gathered}
$$

where $G, K$ and $\eta$ are the shear modulus, the bulk modulus, and the coefficient of viscosity, respectively. Determination of the model parameters can be obtained from laboratory and field testing.

\section{Analytical solutions of settlements using fractional Kelvin-Voigt model}

\subsection{Flamant-Boussinesq elastic solution}

Fig. 2 shows the case where a line force of $p_{0}$ per unit length is applied on the surface of a halfspace. The elastic solution for this plane-strain problem was developed by Flamant (1892) by modifying the three-dimensional solution of Boussinesq (Timoshenko and Goodier 1970). The Flamant-Boussinesq solution using an elastic model (EM) is widely used in foundation engineering for the calculation of stresses and estimation of displacements.

In the polar coordinate system, assume that at a point $\mathrm{P}\left(r_{0}, \pi / 2\right)$ on the ground surface, $\left(u_{\theta}\right)_{\theta=\frac{\pi}{2}, r=r_{0}}=0$, i.e., the influential distance of ground surface settlement is assumed to be $r_{0}$. The displacements at any point in the foundation can be obtained as 


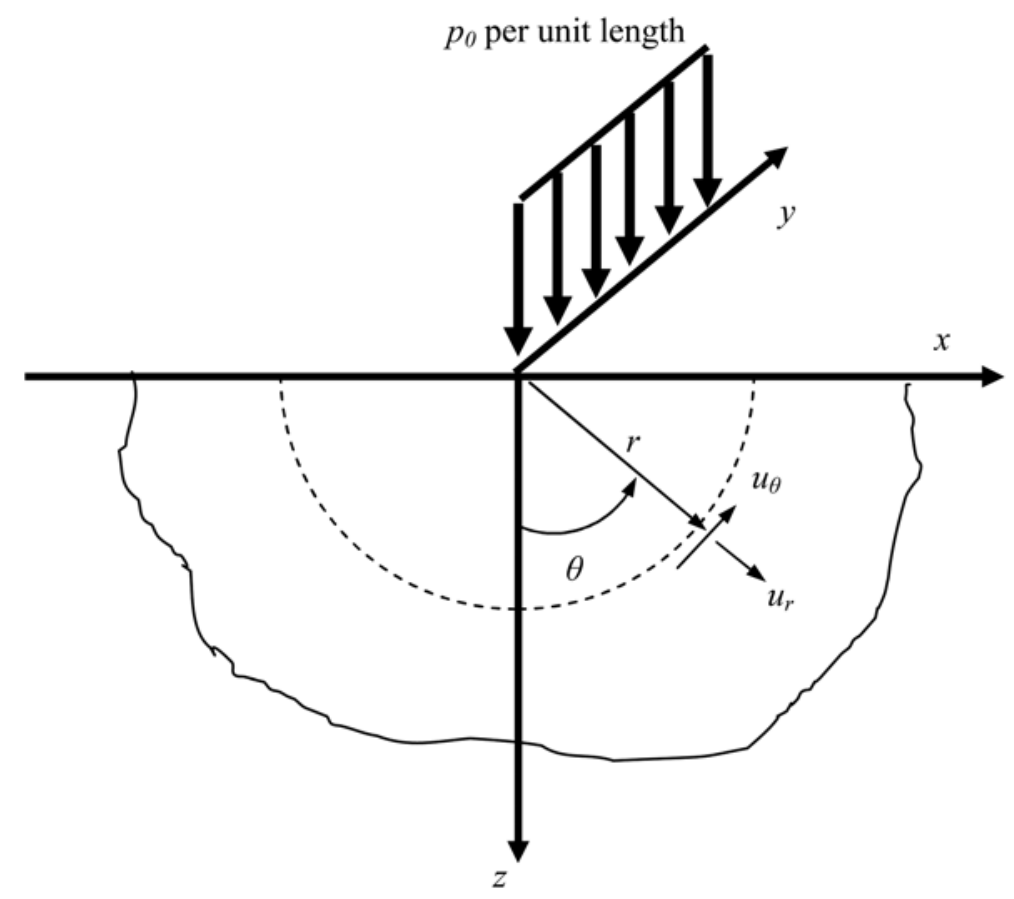

Fig. 2 Schematic illustration of a foundation subjected to vertical line load at the surface

$$
\begin{gathered}
u_{r}=\frac{2 p_{0}\left(1-\mu^{2}\right)}{\pi E} \cos \theta \ln \frac{r_{0}}{r}+\frac{(1-2 \mu)(1+\mu) p_{0}}{\pi E} \theta \sin \theta-\frac{(1+\mu) p_{0}}{\pi E} \cos \theta \\
u_{\theta}=-\frac{2 p_{0}\left(1-\mu^{2}\right)}{\pi E} \sin \theta \ln \frac{r_{0}}{r}+\frac{(1-2 \mu)(1+\mu) p_{0}}{\pi E} \theta \cos \theta
\end{gathered}
$$

where $E$ and $\mu$ are the modulus of elasticity and Poisson's ratio, respectively; $u_{r}$ and $u_{\theta}$ denote the displacements in the radial and circumferential directions, respectively.

Taking $E=144 \mathrm{MPa}, \mu=0.2, p_{0}=1 \mathrm{MPa}$ and $r_{0}=15 \mathrm{~m}$, the distributions of displacements within the soil foundation using the elastic model (EM) are depicted in Fig. 3. It is seen that the surface settlement at the loading point approaches infinity and the settlements within the foundation reduce sharply with increasing depth. For locations far away from the loading point, heave of the foundation occurs.

In the case of $\theta=\pi / 2$, Eqs. (10) and (11) for radial and circumferential displacement components at the ground surface can be given as the following simple form

$$
\begin{gathered}
\left(u_{r}\right)_{\theta=\frac{\pi}{2}}=\frac{(1-2 \mu)(1+\mu) p_{0}}{2 E}=-\frac{3 p_{0}}{4(3 K+G)} \\
\left(u_{\theta}\right)_{\theta=\frac{\pi}{2}}=\frac{2 p_{0}\left(1-\mu^{2}\right)}{\pi E} \ln \frac{r_{0}}{r}=-\frac{p_{0} \ln \frac{r_{0}}{r}}{2 \pi}\left[\frac{3}{(3 K+G)}+\frac{1}{G}\right]
\end{gathered}
$$




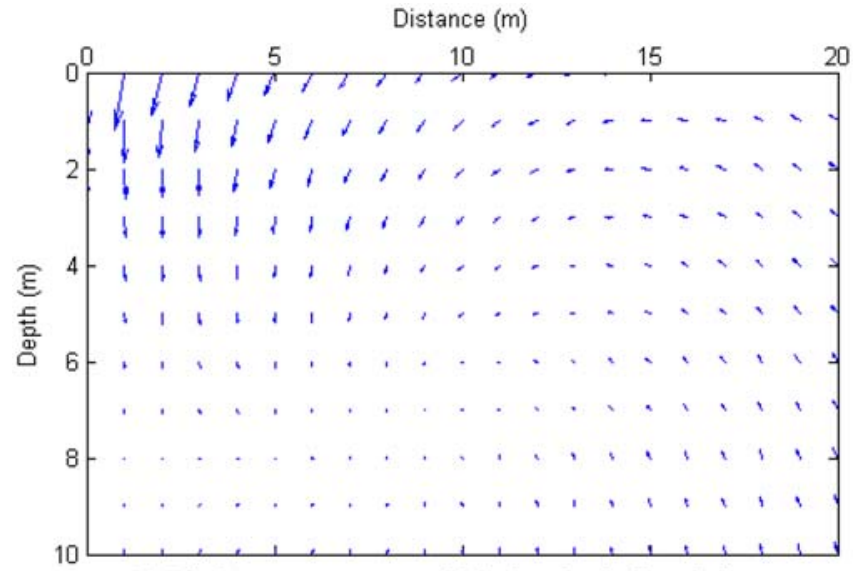

(a) Displacement vectors within the elastic foundation

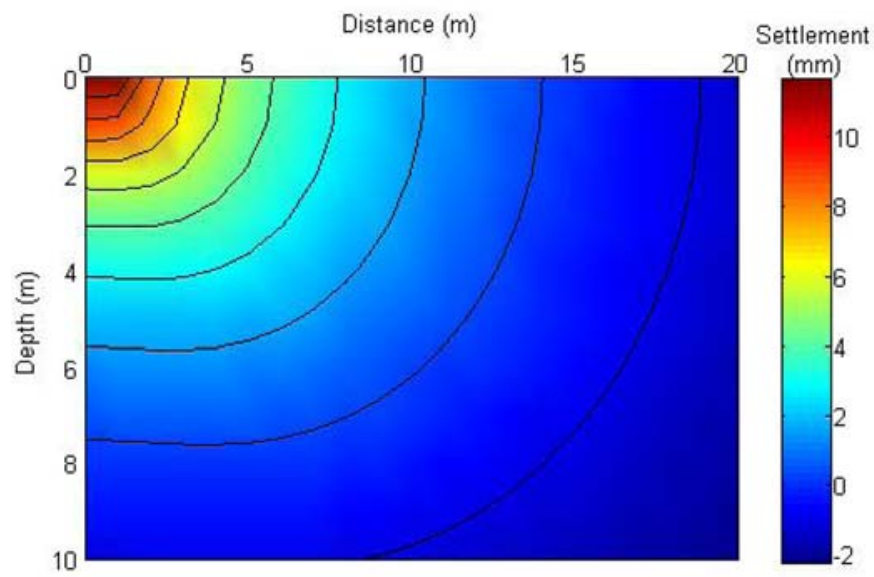

(b) Settlement contour within the elastic foundation

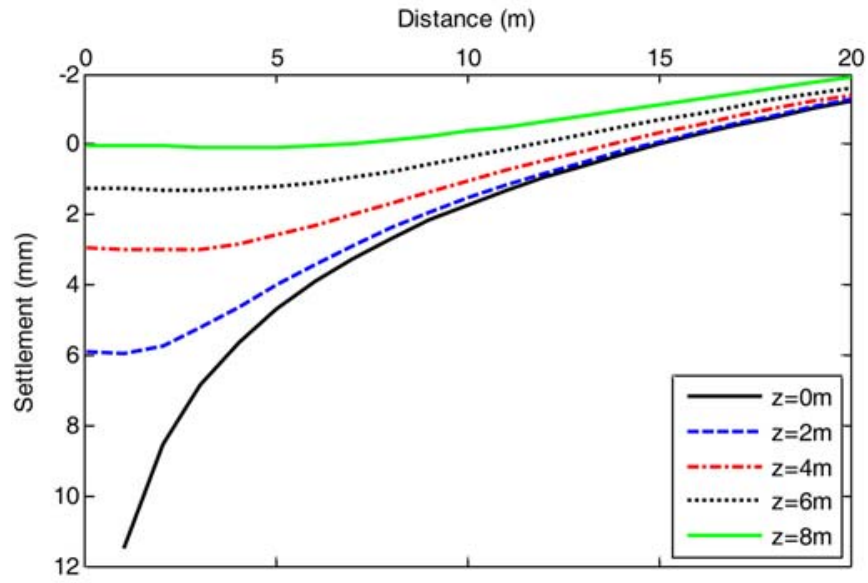

(c) Settlement distributions at different depths

Fig. 3 Displacement distributions calculated from the Flamant-Boussinesq elastic solution 
Eqs. (12) and (13) indicate that there is a logarithmic relationship between the distance to the loading point and the vertical displacement, and that the horizontal displacement at the ground surface is a constant that is not affected by $r$.

\subsection{Viscoelastic solution using classical Kelvin-Voigt model}

Considering a line load applied on the foundation shown in Fig. 2, the loading condition is specified as

$$
p=p_{0} H(t)
$$

where $H(t)$ is the unit-step function defined as $H(t)=\left\{\begin{array}{ll}1 & t \geq 0 \\ 0 & t<0\end{array}\right.$.

To obtain the viscoelastic solution, the volumetric and shear strains of the foundation soil satisfies the KVM. Thus Eqs. (7) and (9) turns into

$$
\begin{aligned}
& Q^{\prime}=G+\eta \frac{d}{d t} \\
& Q^{\prime \prime}=K+\eta \frac{d}{d t}
\end{aligned}
$$

Taking the Laplace transforms of Eqs. (6), (8), (15) and (16), and combining them with Eqs. (12) and (13), the displacements at the ground surface can be expressed by

$$
\begin{gathered}
\left(\bar{u}_{r}\right)_{\theta=\frac{\pi}{2}}=-\frac{3 p_{0}}{4} \frac{1}{(3 K+G+4 \eta s) s} \\
\left(\bar{u}_{\theta}\right)_{\theta=\frac{\pi}{2}}=-\frac{p_{0} \ln \frac{r_{0}}{r}}{2 \pi}\left[\frac{3}{(3 K+G+4 \eta s) s}+\frac{1}{(G+\eta s) s}\right]
\end{gathered}
$$

Taking the inverse Laplace transforms of Eqs. (17) and (18), we get

$$
\begin{gathered}
\left(u_{r}\right)_{\theta=\frac{\pi}{2}}=-\frac{3 p_{0}}{4(3 K+G)}\left(1-e^{-\frac{t}{\tau_{1}}}\right) \\
\left(u_{\theta}\right)_{\theta=\frac{\pi}{2}}=-\frac{p_{0} \ln \frac{r_{0}}{r}}{2 \pi}\left[\frac{3}{(3 K+G)}\left(1-e^{-\frac{t}{\tau_{1}}}\right)+\frac{1}{G}\left(1-e^{-\frac{t}{\tau_{2}}}\right)\right]
\end{gathered}
$$

where $\tau_{1}=4 \eta /(3 K+G), \tau_{2}=\eta / G$. Eqs. (19) and (20) show that the time-dependent displacements are exponential creep functions. It is obvious that if $t \rightarrow \infty$, the ultimate displacements equal the Flamant-Boussinesq solution using elastic model (EM).

\subsection{Viscoelastic solution using fractional Kelvin-Voigt model}

Taking the Laplace transforms of Eqs. (6) to (9) and combined with Eqs. (12) and (13), the 
displacements at the ground surface can be expressed by

$$
\begin{gathered}
\left(\bar{u}_{r}\right)_{\theta=\frac{\pi}{2}}=-\frac{3 p_{0}}{4} \frac{1}{\left(3 K+G+4 \eta s^{\alpha}\right) s} \\
\left(\bar{u}_{\theta}\right)_{\theta=\frac{\pi}{2}}=-\frac{p_{0} \ln \frac{r_{0}}{r}}{2 \pi}\left[\frac{3}{\left(3 K+G+4 \eta s^{\alpha}\right) s}+\frac{1}{\left(G+\eta s^{\alpha}\right) s}\right]
\end{gathered}
$$

In order to take the inverse Laplace transform, note the following characteristics of the fractional derivative in the Laplace transform for zero initial condition

$$
L^{-1}\left[\frac{1}{\left(G+\eta s^{\alpha}\right) s}\right]=\frac{1}{G}\left\{1-E_{\alpha}\left[-\left(\frac{t}{\tau}\right)^{\alpha}\right]\right\}
$$

where $E_{\alpha}$ is the Mittag-Leffler function defined as $E_{\alpha}(t)=\sum_{0}^{\infty} \frac{t^{n}}{\Gamma(1+\alpha n)} ; \tau=\frac{\eta}{G}$.

In view of Eq. (23), taking the inverse Laplace transforms of Eqs. (21) and (22), we get

$$
\begin{gathered}
\left(u_{r}\right)_{\theta=\frac{\pi}{2}}=-\frac{3 p_{0}}{4(3 K+G)}\left\{1-E_{\alpha}\left[-\left(\frac{t}{\tau_{1}}\right)^{\alpha}\right]\right\} \\
\left(u_{\theta}\right)_{\theta=\frac{\pi}{2}}=-\frac{p_{0} \ln \frac{r_{0}}{r}}{2 \pi}\left\{\frac{3-3 E_{\alpha}\left[-\left(\frac{t}{\tau_{1}}\right)^{\alpha}\right]}{(3 K+G)}+\frac{1-E_{\alpha}\left[-\left(\frac{t}{\tau_{2}}\right)^{\alpha}\right]}{G}\right\}
\end{gathered}
$$

where $\tau_{1}=\frac{4 \eta}{3 K+G}, \tau_{2}=\frac{\eta}{G}$.

It is obvious that when $0<\alpha \leq 1, \lim _{t \rightarrow \infty}\left(u_{r}\right)_{\theta=\frac{\pi}{2}}=-\frac{3 p_{0}}{4(3 K+G)}, \lim _{t \rightarrow \infty}\left(u_{\theta}\right)_{\theta=\frac{\pi}{2}}=-\frac{p_{0} \ln \frac{r_{0}}{r}}{2 \pi}\left[\frac{3}{(3 K+G)}+\frac{1}{G}\right]$.

Thus the ultimate displacements equal the elastic solution.

$$
\begin{gathered}
\text { If } \alpha \rightarrow 0, E_{\alpha}(t) \rightarrow \sum_{0}^{\infty} \frac{t^{n}}{\Gamma(1)}=\sum_{0}^{\infty} t^{n}=\frac{1}{1-t}(-1<t<1) \text {. Thus Eqs. (24) and (25) turn into } \\
\left(u_{r}\right)_{\theta=\frac{\pi}{2}}=-\frac{3 p_{0}}{8(3 K+G)} \\
\left(u_{\theta}\right)_{\theta=\frac{\pi}{2}}=-\frac{p_{0} \ln \frac{r_{0}}{r}}{4 \pi}\left[\frac{3}{3 K+G}+\frac{1}{G}\right]
\end{gathered}
$$

Eqs. (26) and (27) indicate that the displacements at the ground surface are not time-dependent any more and the displacement values are half of those from the elastic solution. Therefore, the FKVM collapses to be a ideal elastic model as $\alpha \rightarrow 0$. 
If $\alpha=1, E_{\alpha}(t)=\sum_{0}^{\infty} \frac{t^{n}}{\Gamma(1+\alpha n)}=\sum_{0}^{\infty} \frac{t^{n}}{n !}=e^{t}$. Obviously, Eqs. (23) and (24) currently become the viscoelastic solution, which means that the FKVM turns into a classical KVM.

\section{Numerical example and analysis}

\subsection{Comparison of elastic and viscoelastic models}

How to determine the fractional model parameters is a major concern in practical applications. To carry out a preliminary investigation of these parameters' influence on the calculation results, a parametric study is presented as following. Here, we analyze the quasistatic problem of a viscoelastic foundation subjected to line loading $p_{0}=1 \mathrm{MPa}$ using the FKVM. The basic parameters used in this analysis are listed in Table 1.

Fig. 4 shows the surface settlement results using the fractional model at $t=10 \mathrm{~d}$ and $50 \mathrm{~d}$, together with the results from the classical KVM solution and the Flamant-Boussinesq elastic solution. The present results show that the discrepancy between the results from FKVM and KVM increases as $t$ increases. The calculated settlements using KVM develops rapidly with the elapsed time while those using FKVM will take much more time.

\subsection{Parametric study of the FKVM}

To study the influence of the coefficient of viscosity in the FKVM, $\eta$ is set to 500,1000 and $1500 \mathrm{MPa} \cdot \mathrm{d}$. Fig. 5 depicts the settlement-time curves at $r=1 \mathrm{~m}$ over time using the FKVM, the KVM and the EM. It is shown that the coefficient of viscosity mainly affects the overall creeping rate of the foundation soil. For the same time point, the settlements increase with the decrease of $\eta$. Note that the ultimate settlement in the viscoelastic solution is equal to that in the elastic solution, $\eta$ may determine the magnitude of creeping by controlling the time duration to achieve the ultimate settlement. If $\eta$ decreases, the creeping effect will function more slowly and the ultimate settlement will be obtained for a much longer time. Results from the classical Kelvin-Voigt model $(\alpha=1)$, which is shown in Fig. 6 by a dashed line, shows larger settlements and less time to get to the ultimate settlement.

Table 1 Parameters of the FKVM

\begin{tabular}{cc}
\hline \hline Item & Value \\
\hline Young's modulus $E(\mathrm{MPa})$ & 144 \\
Poisson's ratio $\mu$ & 0.2 \\
Shear modulus $G(\mathrm{MPa})$ & 60 \\
Bulk modulus $K(\mathrm{MPa})$ & 80 \\
Coefficient of viscosity $\eta(\mathrm{MPa} \cdot \mathrm{d})$ & 1000 \\
Fractional differential order $\alpha$ & 0.5 \\
$r_{0}(\mathrm{~m})$ & 15 \\
\hline
\end{tabular}




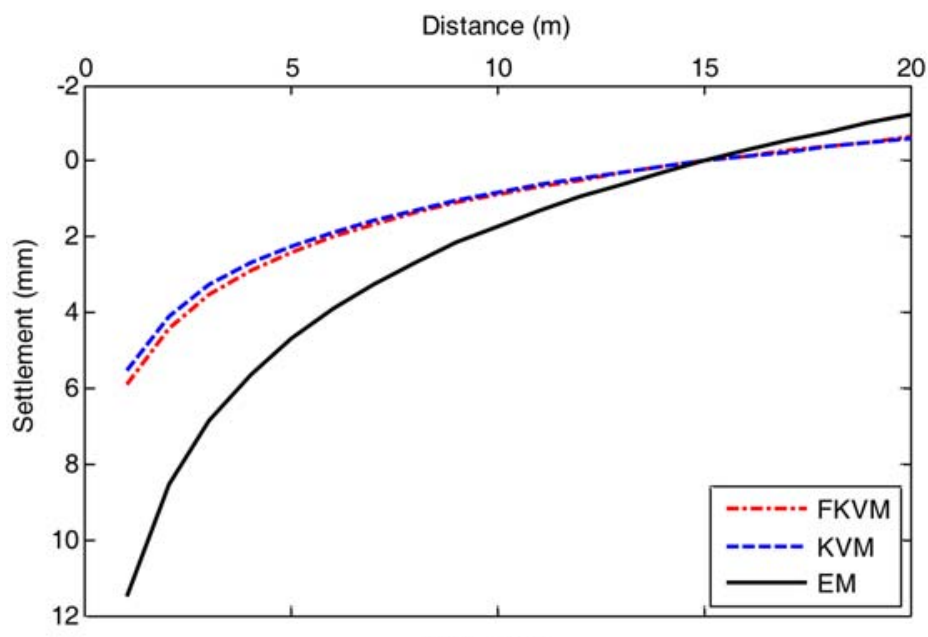

(a) $t=10 \mathrm{~d}$

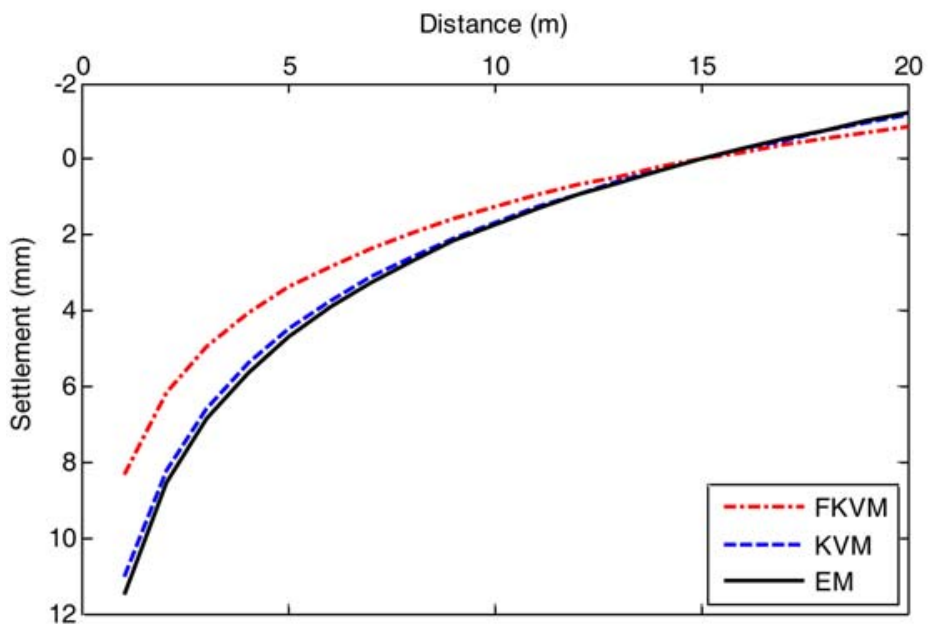

(b) $t=50 \mathrm{~d}$

Fig. 4 Comparsion of settlements calculated using elastic, Kelvin-Voigt and fractional Kelvin-Voigt models

To investigate the influence of the fractional order of differential operator in the FKVM, $\alpha$ is set to $0.1,0.3,0.5,0.7$ and 0.9 while $\eta$ is fixed to $1000 \mathrm{MPa} \cdot \mathrm{d}$. Fig. 6 illustrates the settlement at $r=1$ $\mathrm{m}$ over time. It is shown that the fractional order $\alpha$ has a significant influence on the long-term performance of the foundation surface settlements. At $t_{1 / 2}=10 \mathrm{~d}$, the settlements are half of the ultimate settlement $(5.75 \mathrm{~mm})$ for any value of $\alpha . t_{1 / 2}$ is found to have an approximately linear relationship with $\eta$ and $1 / E$. After that time point, with the increase of $\alpha$, the foundation settlement and corresponding settlement rate increase. Therefore, $\alpha$ may determine the functioning law of creeping effect by separating the time-dependent deformation of soil foundation into two stages. The first stage may associate with the consolidation process of the foundation soil and the second stage is mainly due to secondary compression. Therefore, a small $\alpha$ value is fit for sand foundation, which has a small permeability coefficient. While for soft clay foundation, the $\alpha$ value should be 


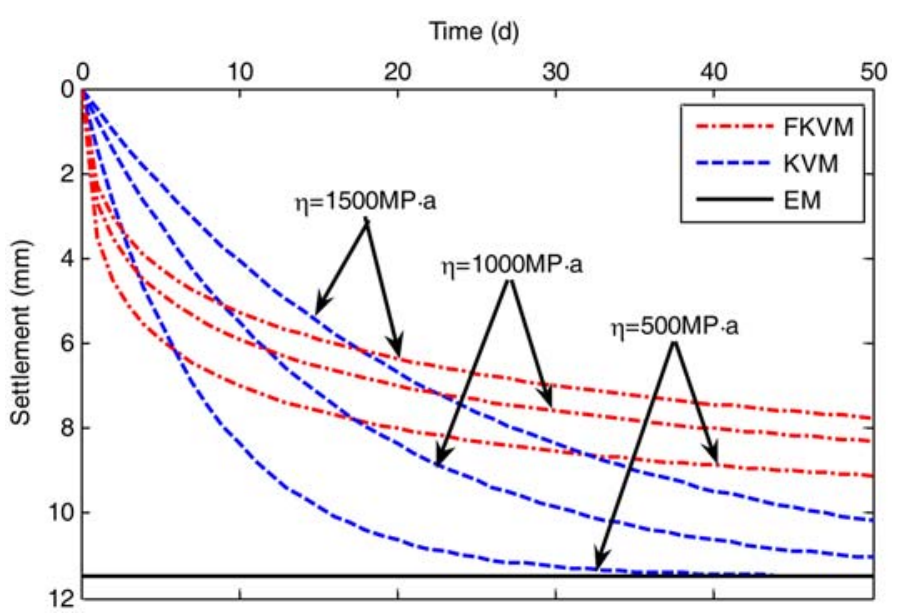

Fig. 5 Settlement-time relationship at $r=1 \mathrm{~m}$ under different $\eta$ values

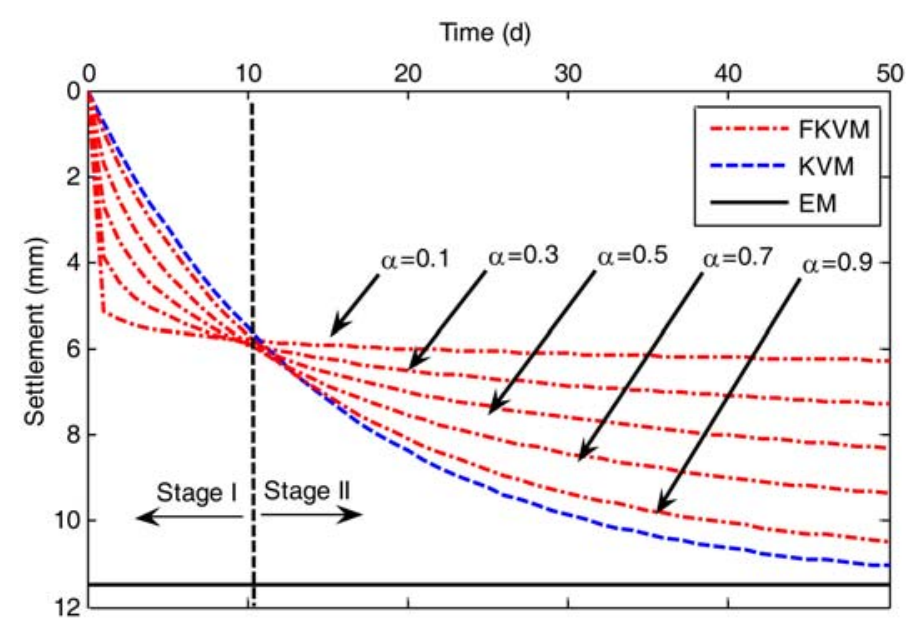

Fig. 6 Settlement-time relationship at $r=1 \mathrm{~m}$ under different $\alpha$ values

increased. In the KVM where $\alpha=1$ (the dashed line in Fig. 6), a minimum settlement rate at stage I and a maximum settlement rate at stage II is obtained.

The key feature of the FKVM illustrated in Figs. 5 and 6 is the capability to represent generalised viscoelastic behaviour over many decades of time with only three parameters. It is also indicated from the above parametric study that the classical viscoelastic model may overestimate the development of long-term settlement (Stage II) and fail to describe the attenuation creep of soil mass accurately. Using the fractional viscoelastic models, we can obtain the best fitting effect of the actual soil deformation characteristics by selecting appropriate values of the fractional differential order and the coefficient of viscosity. In geotechnical design, this model can provide more accurate settlement prediction and thus save construction costs of foundations or embankments. 


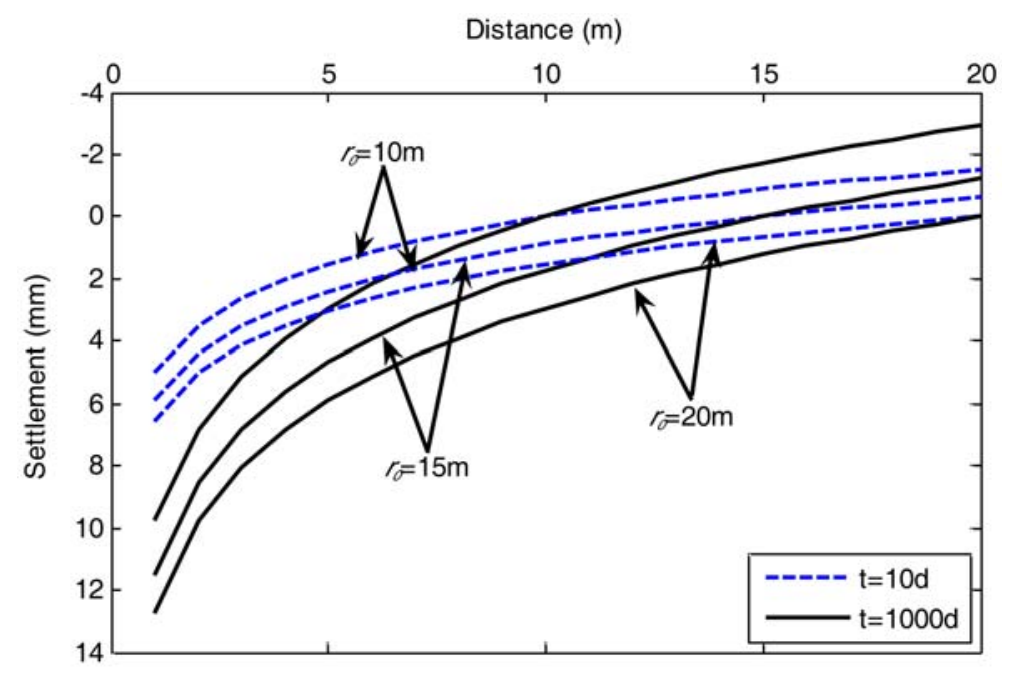

Fig. 7 Settlement distributions at the foundation surface under different predetermined $r_{0}$ values

\subsection{Determination of the influential distance}

The determination of influential distance due to loading is another major engineering concern. Fig. 7 shows the settlement results when the value of $r_{0}$ is set to 10,15 and $20 \mathrm{~m}$ while $\alpha$ is fixed to be 0.5 . It is shown that with the increase of $r_{0}$, the time-dependent and ultimate settlement $(t=1000 \mathrm{~d})$ will increase but the settlement profile is not affected significantly. If the influential distance of settlement is overestimated (like $r_{0}=20 \mathrm{~m}$ ), a larger settlements will be predicted.

\section{Conclusions}

Based on the theory of viscoelasticity and fractional calculus, a fractional Kelvin-Voigt viscoelastic model is developed to account for the time-dependent behaviour of soil foundations under concentrated line load. Analytical solution of displacements in the foundation was derived using Laplace transforms. The fractional theory shows its potential in modeling the long-term foundation deformation. The presented analytical procedure can be extended to solve other geotechnical problems. From a parametric study, the fractional order of derivative differential operator and the coefficient of viscosity exert a significant influence on the time-dependent settlements of foundation. Compared with the KVM, the FKVM can accurately capture the actual deformation of soil foundations under loading.

However, the empirical relationship between the model parameters and soil properties is an important issue to be solved, which requires the support of abundant measurement data from laboratory and field experiments. Careful attention should be paid to the predetermined influential distance, as well. 


\section{Acknowledgements}

The financial supports provided by the National Basic Research Program of China (973 Program) (Grant No. 2011CB710605) and National Key Technology R\&D Program of China (2012BAK10B05) are gratefully acknowledged.

\section{References}

Atanackovic, T. M. and Stankovic B. (2004), "Stability of an elastic rod on a fractional derivative type of foundation", J. Sound Vib., 277(1-2), 149-161.

Bagley, R. L. and Torvik, P. J. (1983), "Fractional calculus-a different approach to the analysis of viscoelastically damped structures", Am. Inst. Aeronaut. Astronaut. J., 21(5), 741-748.

Bagley, R. L. and Torvik, P. J. (1986), "On the fractional calculus model of viscoelastic behavior", J. Rheol, 30(1), 133-155.

Bjerrum, L. (1967), "Engineering geology of Norwegian normally-consolidated marine clays as related to settlement of buildings", Géotechnique, 17(2), 81-118.

Christie, I. F. (1964), “A re-appraisal of Merchant's contribution to the theory of consolidation”, Géotechnique, 14(4), 309-320.

Dikmen, Ü. (2005), "Modeling of seismic wave attenuation in soil structures using fractional derivative scheme", J. Balkan Geophys. Soc., 8(4), 175-188.

Gemant, A. (1936), "A method of analyzing experimental results obtained from elasto-viscous bodies", J. Appl. Phys., 7, 311-317.

Justo, J. L. and Durand, P. (2000), "Settlement-time behaviour of granular embankments", Int. J. Numer. Anal. Meth. Geomech., 24(3), 281-303.

Kaliakin, V. N. and Dafalias, Y. F. (1990), "Theoretical aspects of the elastoplastic-viscoplastic bounding surface model for cohesive soils", Soil Found., 30(3), 11-24.

Li, G. G., Zhu, Z. Y. and Cheng, C. J. (2001), "Dynamical stability of viscoelastic column with fractional derivative constitutive relation", Appl. Math. Mech. -Engl. Ed., 22(3), 294-303.

Liu, L. C., Yan, Q. F. and Sun, H. Z. (2006), "Study on model of rheological property of soft clay", Rock Soil Mech., 27(S1), 214-217. (in Chinese)

Miller, K. S. and Ross, B. (1993), An Introduction to the Fractional Calculus and Fractional Differential Equations, Wiley, New York .

Padovan, J. (1987), "Computational algorithms for FE formulations involving fractional operators", Comput. Mech., 2(4), 271-287.

Paola, M. Di., Marino, F. and Zingales M. (2009), "A generalized model of elastic foundation based on longrange interactions: Integral and fractional model”, Int. J. Solids Struct., 46(17), 3124-3137.

Rossikhin, Y. A. and Shitikova M. V. (2010), "Application of fractional calculus for dynamic problems of solid mechanics: novel trends and recent results", Appl. Mech. Rev., 63(1), 010801, 1-52.

Timoshenko, S. P. and Goodier, J. N. (1970), Theory of Elasticity, McGraw-Hill, New York.

Welch, S. W. J., Rorrer, R. A. L. and Ronald, G. D. Jr. (1999), "Application of time-based fractional calculus methods to viscoelastic creep and stress relaxation of materials", Mech. Time-Depend. Mater., 3(3), 279-303.

Yin, J. H. and Graham, J. (1994), "Equivalent times and one-dimensional elastic viscoplastic modelling of timedependent stress-strain behaviour of clays", Can. Geotech. J., 31(1), 42-52.

Zhang, W. and Shimizu, N. (1998), "Numerical algorithm for dynamic problems involving fractional operators", J. Soc. Mech. Eng. Int. J. Ser. C., 41(3), 364-370.

Zhu, H. H., Liu, L. C. and Ye, X. W. (2011), "Response of a loaded rectangular plate on fractional derivative viscoelastic foundation", J. Basic Sci. Eng., 19(2), 271-278. (in Chinese)

$C C$ 